\title{
Raman and FTIR Microscopy on a Single Microscope: Demonstration of the Synergism of Collecting Complementary Vibrational Spectra from the Same Spot
}

\author{
F. Adar*, G. LeBourdon**, J. Reffner***, and A. Whitley* \\ * Jobin Yvon, Inc., 3880 Park Ave., Edison, N.J. \\ * Jobin Yvon, SA, Villeneuve d'Ascq, France \\ * SensIR, Danbury, CT. 06810
}

Raman and Infrared spectroscopy are two photonic techniques providing information on vibrational transitions in molecules and crystals. They have been well exploited over the past 40 years in a variety of applications. Most analytical laboratories have multiple FTIR instruments reflecting the level of development of this tool. Until the last 10 years, Raman has tended to be a more research-type tool. However, enabling technologies, including the microscope, multichannel detectors, air-cooled lasers, holographic notch filters, and inexpensive computers with powerful software, have made Raman instruments smaller, faster and easier to use. Consequently more Raman instruments are appearing in industrial analytical laboratories along side FTIR instruments. The trained analyst will usually select one tool of the two to best fit the requirements of a particular sample. While both techniques detect molecular vibrations, the complementarity of the two is used to select the "better" method of analysis. However, the simultaneous availability of the two can provide a synergism over separate measurements. The origin of this synergism will be discussed with examples. In order for a combination Raman/FTIR microscope to be of maximum benefit, the combination of the two should not subtract from the performance of either. To achieve this, a very small FTIR interferometer was required. The design of the interferometer itself has been optimized for use with a microscope. That is, a typical microscope pupil is of the order of $5-10$ $\mathrm{mm}$, so the interferometer optics have been designed with this dimension in mind. In addition, it was noted that it is rare that infrared (IR) spectra of condensed phase material are sharper than 5-8 $\mathrm{cm}^{-1}$. The travel of the moving mirror was limited to the requirement for $4 \mathrm{~cm}^{-1}$ spectral resolution. The final effect of these two design parameters was to reduce the size of the entire interferometer to 5.5 inches.

Two microscope objectives, optimized for microscopic use, have been designed. The Schwarzschild all-reflecting objective can be used for specular- and diffuse-reflecting samples. Software algorithms are used to convert the recorded acquisitions to standard spectra using respectively Kramers-Kronig and Kubulka-Monk transformations. An objective designed for Attenuated Total Reflection (ATR) has also been optimized for routine analysis. The ATR element is $\mathrm{ZnSe}$, but it is capped with a thin Diamond tip. Because the indices of refraction of these two materials are almost exactly equal, the presence of the diamond does not interfere with the optical properties of the objective. But it does have the effect of making a much more robust element. In order to measure a spectrum with an ATR element, the sample must be brought into contact with the diamond tip. After each measurement the tip is wiped to remove any material from the sample. With a bare ZnSe element, the tip can be scratched, whereas a diamond tip is very robust and ideally suited for repeated usage. Consequently the sample can be viewed through the ATR element. Signal treatment for the ATR objective involves only a simple correction to compensate for the optical penetration depth as a function of wavelength. 

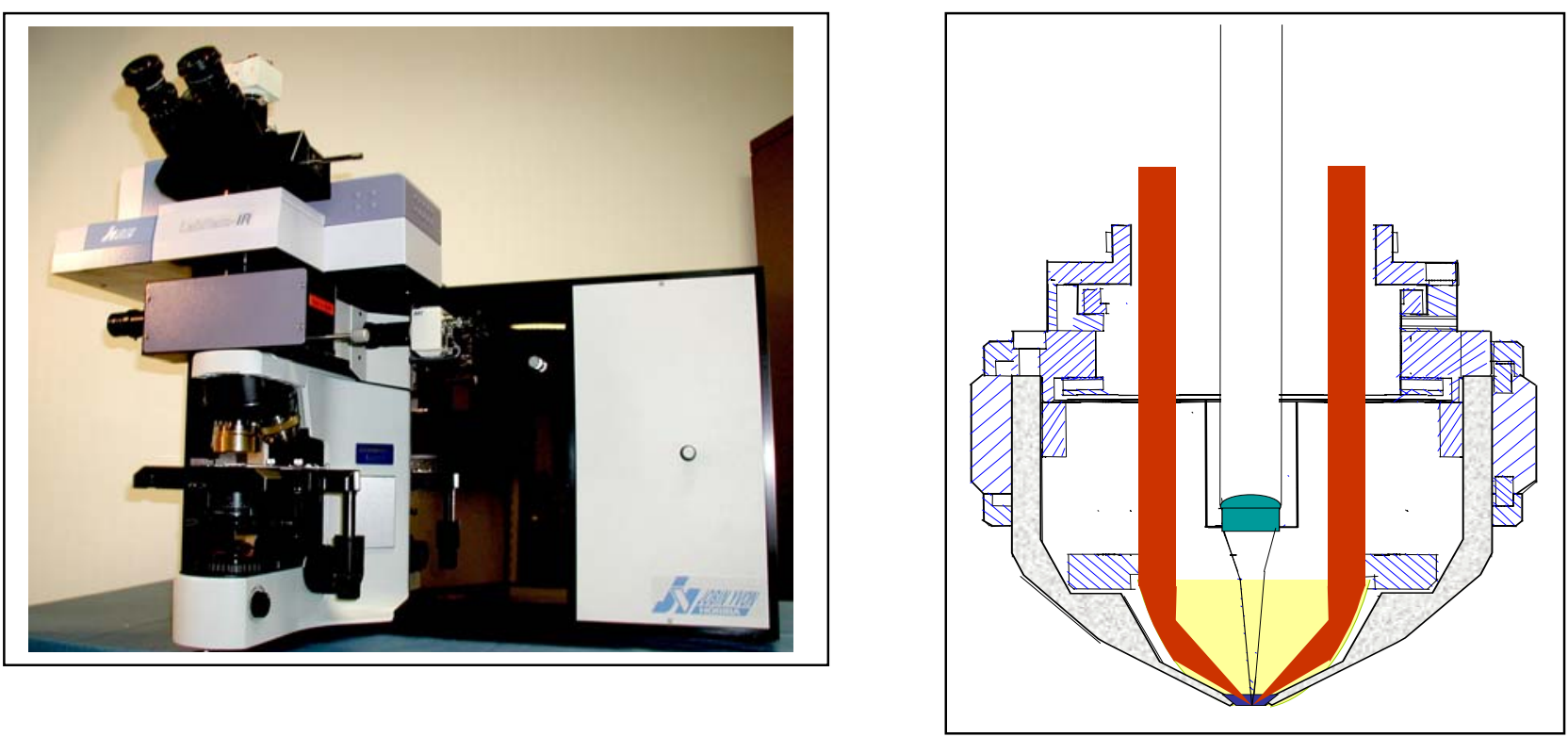

FIG. 1. LabRam with IlluminatIR mounted above microscope (left) and close up of ATR objective (right).

A series of measurements will be shown to illustrate the power of this combination. For example, there will be cases where the high sensitivity of the IR will enable identification or detection of thin surface layers when Raman would fail. Raman, on the other hand, will easily identify metal oxides (with bands down to $100 \mathrm{~cm}^{-1}$ or less) and other inorganics (ie., phosphates, carbonates, etc.) used as additives for various applications in organic matrices. Raman can be used for depth profiling, often without cross-sectioning. Infrared may detect organics adsorbed at low concentrations in inorganics (such as clays or other inorganic materials).

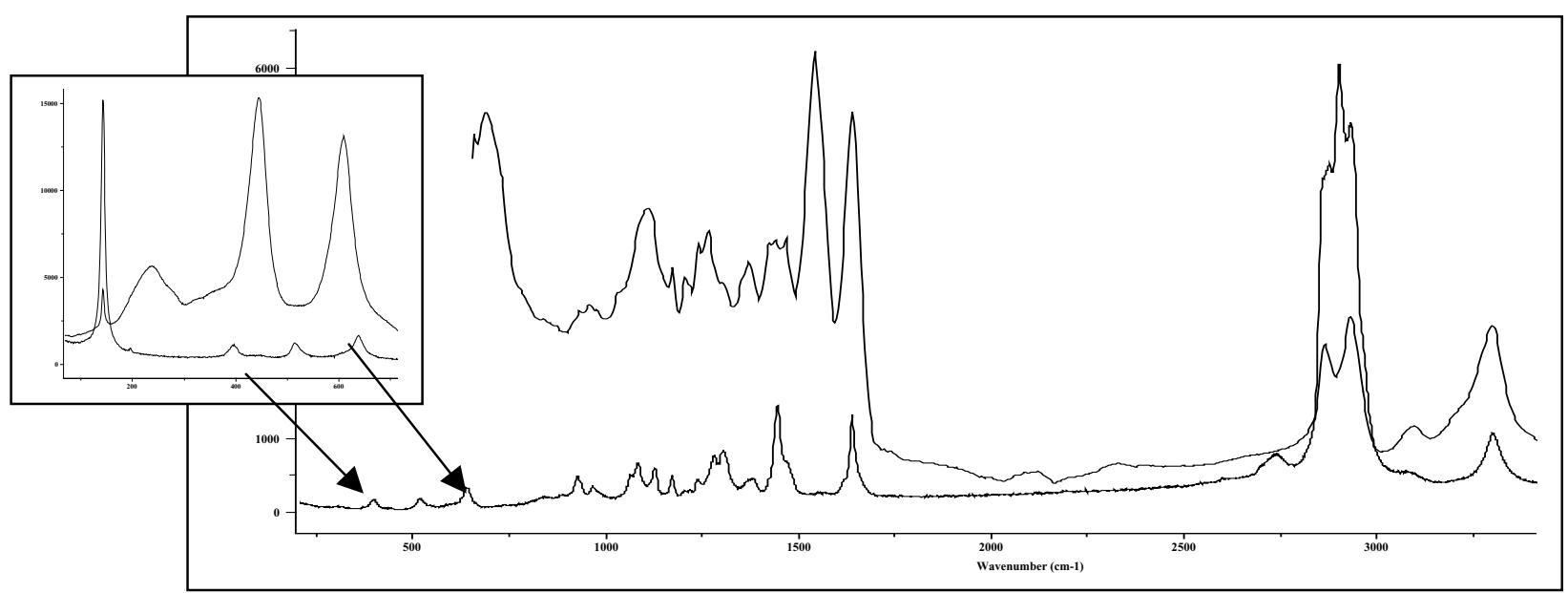

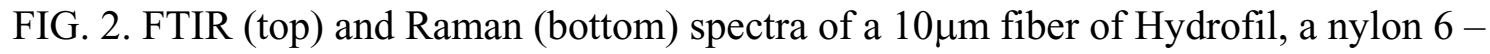
polyethylene glycol (PEG) block copolymer. The inset shows spectra of two common forms of $\mathrm{TiO}_{2}$ pigment, anatase and rutile. Comparison of IR and Raman spectra of fiber indicates the differences in sensitivities to different functional groups of the polymer. Comparison of Raman spectra identifies the pigment as anatase. 